\title{
Evaluation de l'apport de la méthode d'observation des spermatozoïdes à fort grossissement en ICSI
}

\author{
Nathalie SERMONDADE, François VIALARD, Marianne BERGERE, Ibrahim HAMMOUD, \\ Patrick CAVELOT, Jacqueline SELVA, Martine ALBERT
}

Laboratoire d'Histologie - Embryologie - Biologie de la Reproduction et Cytogénétique, Centre Hospitalier Intercommunal de Poissy Saint-Germain-en-Laye, France

\begin{abstract}
RESUME
Nous avons souhaité évaluer l'intérêt de la sélection des spermatozoïdes par la méthode d'observation à fort grossissement, appelée MSOME (Motile Sperm Organelle Morphology Examination), par rapport à celle habituellement réalisée en ICSI. Notre objectif était de déterminer le pourcentage de spermatozoïdes sélectionnés en ICSI conventionnelle qui s'avéraient anormaux à fort grossissement, et d'évaluer la valeur prédictive de ce paramètre sur l'issue de la tentative d'ICSI.

L'étude a concerné 25 tentatives d'ICSI sans sélection d'indication. Pour chaque patient, 25 spermatozoïdes mobiles et "injectables" d'après l'évaluation morphologique conventionnelle en ICSI ont été observés en MSOME à un grossissement supérieur à $\times \mathbf{4 5 0 0}$ et classés selon une grille de lecture adaptée des travaux de Bartoov et de la classification de David modifiée. Nous avons comparé les résultats de MSOME et l'issue des ICSI correspondantes.
\end{abstract}

Dans cette courte série d'ICSI sans sélection d'indication, nous avons retrouvé des fréquences élevées d'anomalies (supérieures à $70 \%$ ) et notamment de vacuoles nucléaires. Nous n'avons pas retrouvé de valeur prédictive de la morphologie spermatique évaluée à fort grossissement, y compris des vacuoles, pour le taux de fécondation, la qualité embryonnaire ou l'issue de I'ICSI. De plus, des grossesses ont été obtenues avec des spermes très altérés. Dans cette série, les vacuoles ne semblent pas avoir de signification péjorative pour l'obtention d'une grossesse.

Plusieurs questions sont soulevées à l'issue de ce travail : quel est le support et la signification des vacuoles observées en MSOME ? Y a-t-il une corrélation entre la morphologie spermatique et le contenu génétique ? Enfin, des études prospectives randomisées semblent nécessaires afin de valider les indications potentielles de cette technique.

Mots clés : morphologie spermatique, observation et sélection des spermatozoïdes à fort grossissement, ICSI, MSOME, IMSI, vacuoles nucléaires

\section{INTRODUCTION}

La morphologie spermatique est considérée par beaucoup d'auteurs comme un très bon facteur prédictif de succès en fertilité naturelle [20,34], en insémination intra-utérine [25] ainsi qu'en fécondation in vitro conventionnelle (FIV) [14, 23, 24, 29]. A l'inverse, il n'a pas été mis en évidence de relation entre les taux de formes normales évalués par le spermocytogramme et les taux de succès globaux en FIV avec injection intracytoplasmique de spermatozoïde (ICSI) [14, 26], exceptés dans les cas de tératospermies totales ou syndromiques touchant principalement la tête spermatique, telles que la macrocéphalie ou la globozoospermie [17, 19, 22]. Plusieurs publications récentes retrouvent néanmoins des taux d'implantation et de grossesses diminués dans les cas où seuls des spermatozoïdes morphologiquement anormaux sont disponibles lors de la micro-injection [16, 17, 19, 22].

Correspondance :

Dr Nathalie SERMONDADE - Laboratoire d'Histologie -

Embryologie - Biologie de la Reproduction et Cytogénétique, Centre Hospitalier Intercommunal de Poissy Saint-Germainen-Laye, 10 rue du Champ Gaillard, 78300 Poissy, France Tel +33139275155 - Fax +33139274425 -

Email nsermondade@hotmail.com 
De plus, certains échecs répétés de FIV ou d'ICSI seraient liés à un effet paternel négatif de spermatozoïdes altérés sur le développement embryonnaire précoce [33].

Or, certains spermatozoïdes apparaissant normaux lors de la sélection en ICSI réalisée au grossissement $\times 200$ 400 pourraient en fait présenter des anomalies structurales qui ne seraient détectées qu'à un plus fort grossissement.

Bartoov et al. [3] ont donc développé la méthode du MSOME (Motile Sperm Organelle Morphology Examination) qui permet une évaluation à très fort grossissement $(x 6000)$ et en temps réel de la morphologie des spermatozoïdes mobiles.

Les premiers résultats publiés d'ICSI avec pré-sélection des spermatozoïdes en MSOME (Intracytoplasmic Morphologically Selected sperm Injection ou IMSI) retrouvent une augmentation significative des taux d'implantation et de grossesses ainsi qu'une diminution du taux de fausse-couche spontanée lorsque le cycle de micro-injection se fait avec des spermatozoïdes sélectionnés à fort grossissement par rapport aux cycles précédents réalisés sans sélection [3], ou par rapport à des couples appariés avec cycle d'ICSI conventionnelle [4], ou par rapport à un groupe où seuls des spermatozoïdes de "second choix " sont disponibles pour l'injection [9]. De plus, une augmentation des taux d'implantation et de grossesses a été rapportée dans les cas d'échecs d'implantation après ICSI [21]. La morphologie évaluée à un grossissement supérieur à x6000 semblerait donc constituer un facteur important de succès après ICSI.

Cependant, la validation des applications potentielles de la méthode est encore en cours, puisque aucun essai randomisé n'a été publié à ce jour.

Le premier objectif de notre étude a été de standardiser la méthode et de proposer une grille de lecture des spermatozoïdes en MSOME. Le deuxième a été d'évaluer l'apport de la méthode d'observation des spermatozoïdes à fort grossissement par rapport à la sélection classique effectuée en ICSI. Pour cela, nous avons déterminé, dans une série de patients pris en charge en ICSI, le pourcentage de spermatozoïdes « injectables » au grossissement x200-400 (" normaux " ou " les plus normaux possible " lorsqu'il n'y avait pas de spermatozoïde normal disponible) qui s'avéraient être anormaux à fort grossissement. Les résultats de l'ICSI classique ont été recueillis afin d'évaluer l'impact de la morphologie des spermatozoïdes observés à fort grossissement sur les taux de fécondation, la qualité embryonnaire et l'issue de la tentative d'ICSI.

\section{MATÉRIEL ET MÉTHODES}

\section{Patients}

L'étude a concerné 25 tentatives d'ICSI avec sperme frais, sans sélection particulière parmi les indications d'ICSI, chez des couples pris en charge dans le service de Biologie de la Reproduction du Centre Hospitalier de Poissy entre mars et mai 2006 (Groupe A). Les fréquences des anomalies des spermatozoïdes en MSOME du groupe A ont été comparées à celles de 5 hommes témoins ayant des paramètres spermatiques normaux et ayant fait preuve de leur fertilité (Groupe B).

Ont été exclus de l'étude les tentatives d'ICSI réalisées dans le cadre d'un don d'ovocyte, celles réalisées avec des spermatozoïdes congelés et/ou prélevés chirurgicalement ou dans des cas d'oligozoospermie extrême $(<0,01 \mathrm{M} / \mathrm{mL})$, ainsi que les tentatives pour lesquelles moins de 2 ovocytes injectables étaient recueillis.

Les vingt-cinq tentatives d'ICSI sans sélection d'indication comprennent 21 ICSI de première intention pour oligoasthénotératospermie (OAT) selon les critères de l'OMS [36], et 4 ICSI pour échec de fécondation en FIV conventionnelle.

\section{Préparation du sperme}

Après liquéfaction, les paramètres conventionnels du spermogramme ont été évalués conformément aux recommandations de l'OMS [36]. Le sperme a été préparé par migration sur gradient de densité 2 couches (PureSperm ${ }^{\circledR} 100$, Nidacon). La majorité de la suspension obtenue a été utilisée pour l'ICSI. La fraction restante a permis l'évaluation de la numération et de la mobilité en microscopie photonique et par analyse automatisée du sperme (CASA) (Human Motility 12.2L, Hamilton Thorne Biosciences), la réalisation d'un spermocytogramme et l'observation à fort grossissement de la morphologie spermatique.

\section{Déroulement de I'ICSI}

Les FIV avec microinjection se sont déroulées selon les protocoles habituellement utilisés dans le laboratoire. Le score cumulé embryonnaire de Steer [32] a été déterminé à $\mathrm{J} 2$ pour évaluer la qualité des embryons transférés. Un à trois embryons ont été transférés à $\mathrm{J} 2$ (ou à J3) et les éventuels embryons surnuméraires ont été congelés selon la procédure habituelle à l'aide d'un Minicool 40PC.

\section{Spermocytogramme}

La morphologie spermatique sur sperme migré a été évaluée pour chaque patient le jour de la tentative selon la méthode de David modifiée $[1,15]$. 


\section{Observation à fort grossissement de la morpho- logie spermatique}

Un échantillon de $2 \mu \mathrm{L}$ de spermatozoïdes migrés a été dilué dans une micro-goutte de $4 \mu \mathrm{L}$ de polyvinyl pyrolidone (PVP Medium, Medicult) et placé dans une boîte WillCo-dish ${ }^{\circledR}$ (WillCoWells) à fond en verre d'épaisseur $0,17 \mathrm{~mm}$ pour observation.

L'examen a été réalisé avec un microscope inversé Nikon ${ }^{\circledR}$ TE2000-U équipé d'un objectif Nomarsky x100 à l'immersion. La morphologie spermatique a été évaluée avec un grossissement compris entre 4000 et 10000 fois (objectif $\times 100$, rapport caméra/moniteur $\times 30$, lentille facultative $\times 1,5$ et zoom compris entre $\times 0,9$ et $\times 2,25$ ).

Vingt-cinq spermatozoïdes mobiles de la fraction migrée restant disponibles après ICSI et " injectables " selon l'observation en ICSI classique ont été observés à très fort grossissement et classés selon la grille de lecture proposée (Figure 1).

\section{Evaluation de l'apport de la méthode d'observation des spermatozoïdes à fort grossissement}

Les résultats de l'analyse en MSOME ont été comparés avec ceux du spermocytogramme après migration, et les résultats de l'ICSI ont été corrélés aux résultats de l'observation en MSOME.

\section{Analyses statistiques}

Les résultats ont été analysés à l'aide du logiciel StatView 5.0 (Windows). Ils sont exprimés en valeur moyenne \pm écart-type. Les tests statistiques utilisés ont été le test de Mann Whitney, le test de corrélation de Spearman et le test de Khi2, avec un seuil de significativité fixé à $p<0,05$.

\section{RÉSULTATS}

\section{Description des tentatives et des paramètres spermatiques conventionnels}

Les paramètres spermatiques conventionnels des groupes $A$ et $B$ sont présentés dans le Tableau 1.

Les résultats des tentatives d'ICSI classique correspondant à l'observation en MSOME sont présentés dans le Tableau 2. Les 25 transferts ont permis d'initier 6 grossesses, soit un taux de grossesse global par transfert de $24 \%$.

Si l'on compare les tentatives ayant abouti ou non à une grossesse clinique dans le Groupe A, il n'y a pas de différence significative entre les deux sous-groupes en ce qui concerne l'âge des hommes et femmes, le rang moyen de la tentative, les taux de fécondation et de clivage. Les nombres d'ovocytes recueillis et d'ovocytes matures en métaphase II sont supérieurs dans le groupe
"Grossesse " par rapport au groupe " Pas de grossesse ", mais sans que cette différence n'atteigne la significativité. Par contre, le nombre moyen de zygotes 2 PN obtenus à $\mathrm{J} 1$, le nombre moyen d'embryons diploïdes obtenus à $\mathrm{J} 2$ et le nombre moyen d'embryons congelés sont significativement supérieurs dans le groupe "Grossesse " par rapport au groupe " Pas de grossesse ". On ne retrouve pas de différence significative de la qualité embryonnaire des embryons transférés évaluée par le score de Steer [32] (Tableau 3).

\section{Mise au point technique}

Dans notre grille de lecture, la plupart des critères proposés par Bartoov [5] ont été utilisés tels que, et d'autres ont été adaptés (Figure 2). Deux critères nous semblant difficilement observables ont été supprimés : le critère "Mitochondrie " (cette structure ne nous semblant pas visualisable en MSOME) et le critère " Acrosome " (les limites et la normalité de cette structure nous semblant impossibles à déterminer).

La grille de lecture proposée (Figure 1) est ainsi inspirée à la fois de la grille de lecture de Bartoov [5] et de la classification de David modifiée $[1,15]$. Trois critères concernant la tête et la pièce intermédiaire (Figure 3, photos b et $\mathbf{c}$ ), ainsi que la catégorie "Vacuoles " (Figure 3, photo d) selon la classification de Bartoov [5], ont été ajoutés à la classification de David modifiée.

La définition du spermatozoïde normal (Figure 3, photo a) en MSOME reprend celle proposée par Bartoov :

- Taille : longueur $=4,75 \pm 0,28 \mu \mathrm{m}$ et largeur $=3,28$ $\pm 0,20 \mu \mathrm{m}$;

- Tête ovale, lisse et symétrique ;

- Absence de vacuole nucléaire ou présence d'une seule vacuole nucléaire représentant au maximum $4 \%$ de la surface de la tête ;

- Absence d'anomalie de la pièce intermédiaire et de la pièce principale du flagelle.

\section{Evaluation de la morphologie spermatique en MSOME}

Pour chaque patient ou témoin, l'évaluation de la morphologie des spermatozoïdes à fort grossissement a été réalisée sur 25 spermatozoïdes " injectables », c'est-à-dire présélectionnés à faible grossissement (x200-400) et considérés comme utilisables en ICSI.

Les fréquences des anomalies spermatiques observées dans la population témoin (soient 125 spermatozoïdes) et dans la population étudiée (soient 625 spermatozoïdes) sont présentées dans le Tableau 4. 
Tableau 1 : Paramètres spermatiques avant et après migration des patients étudiés.

\begin{tabular}{cccc}
\hline & Volume (mL) & $\begin{array}{c}\text { Groupe A } \\
(\mathbf{n}=\mathbf{2 5})\end{array}$ & $\begin{array}{c}\text { Groupe B } \\
(\mathbf{n}=5)\end{array}$ \\
\hline \multirow{2}{*}{$\begin{array}{c}\text { Spermo- } \\
\text { gramme de } \\
\text { référence sur } \\
\text { sperme entier }\end{array}$} & Concentration (M/mL) & $22,6 \pm 1,5$ & $3,9 \pm 1,8$ \\
\cline { 2 - 4 } & Mobilité a+b (\%) & $27,5 \pm 12,9$ & $51,0 \pm 17,5$ \\
\cline { 2 - 4 } & \% de formes typiques & $12,0 \pm 10,0$ & $14,8 \pm 7,7$ \\
\hline \multirow{2}{*}{$\begin{array}{c}\text { Sperme migré } \\
\text { le jour de la } \\
\text { tentative }\end{array}$} & Moncentration (M/mL) & $18,8 \pm 15,1$ & NR \\
\cline { 2 - 4 } & Mobilité a+b (\%) & $42,0 \pm 19,7$ & NR \\
\hline
\end{tabular}

Groupe $A$ : patients pris en charge en ICSI.

Groupe $B$ : témoins ; Valeur moyenne \pm écart-type : NR : non réalisé.
Tableau 2 : Description et résultats des tentatives d'ICSI.

\begin{tabular}{|c|c|}
\hline Nombre moyen d'ovocytes recueillis ${ }^{\text {a }}$ & $11,64 \pm 6,33$ \\
\hline Nombre moyen d'ovocytes en $\mathrm{M} \|^{\mathrm{a}}$ & $8,08 \pm 5,18$ \\
\hline Nombre moyen de zygotes $(2 \mathrm{PN})^{\mathrm{a}}$ & $6,20 \pm 3,93$ \\
\hline Taux de fécondation $(\%)^{\mathrm{a}}$ & $77,2 \pm 19,0 \%$ \\
\hline Nombre moyen d'embryons diploïdes ${ }^{a}$ & $6,08 \pm 3,89$ \\
\hline Taux de clivage $(\%)^{a}$ & $98,4 \pm 4,5 \%$ \\
\hline Nombre d'embryons transférés ${ }^{a}$ & $1,96 \pm 0,45$ \\
\hline Nombre d'embryons congelés ${ }^{a}$ & $3,24 \pm 3,61$ \\
\hline Taux de grossesse clinique par transfert (\%) & $24 \%(6 / 25)$ \\
\hline Taux d'implantation (\%) & $14,3 \%(7 / 49)$ \\
\hline Taux de grossesse multiple (\%) & $16,7 \%(1 / 6)$ \\
\hline Taux de fausse-couche spontanée (\%) & $16,7 \%(1 / 6)$ \\
\hline
\end{tabular}

${ }^{a}:$ valeur moyenne \pm écart-type

Tableau 3 : Comparaison des paramètres des tentatives en fonction de I'issue des ICSI.

"Grossesse " «Pas de grossesse " p

\begin{tabular}{lccc}
\hline Nombre de cycles & 6 & 19 & \\
Age moyen de la patiente & $31.3 \pm 2.8$ & $32.6 \pm 4.9$ & NS \\
Age moyen du patient & $35.7 \pm 2.4$ & $35.4 \pm 5.4$ & NS \\
Rang moyen de la tentative & $1.2 \pm 0.4$ & $1.8 \pm 1.0$ & NS \\
\hline Nombre moyen d'ovocytes recueillis & $16.2 \pm 8.2$ & $10.2 \pm 5.3$ & NS \\
Nombre moyen d'ovocytes en M II & $12.5 \pm 7.7$ & $6.7 \pm 3.3$ & NS \\
Nombre moyen de zygotes (2PN) & $9.8 \pm 5.1$ & $5.1 \pm 2.7$ & $\mathbf{0 . 0 3 3}^{\star}$ \\
Taux de fécondation (\%) & $83.7 \pm 10.4$ & $75.2 \pm 20.8$ & NS \\
Nombre moyen d'embryons diploïdes & $9.7 \pm 5.2$ & $4.9 \pm 2.7$ & $\mathbf{0 . 0 3 9 ^ { * }}$ \\
Taux de clivage (\%) & $97.9 \pm 0.5$ & $98.5 \pm 0.4$ & NS \\
Nombre d'embryons transférés & $1.8 \pm 0.4$ & $2.0 \pm 0.5$ & $\mathrm{NS}$ \\
Score cumulé embryonnaire de Steer & $28.67 \pm 10.25$ & $23.21 \pm 8.72$ & $\mathrm{NS}$ \\
Nombre d'embryons congelés & $7.0 \pm 4.0$ & $2.0 \pm 2.6$ & $\mathbf{0 . 0 1 1 ^ { * }}$ \\
\hline
\end{tabular}

Valeur moyenne \pm écart-type. ${ }^{*}=$ significatif. $N S=$ non significatif. 
Tableau 4 : Fréquences des anomalies observées dans les populations étudiées chez les 25 patients ( $n=625$ spermatozoïdes) et chez les 5 témoins ( $n=125$ spermatozoïdes).

\begin{tabular}{|c|c|c|c|}
\hline $\begin{array}{c}\text { Détail des anomalies } \\
\text { en MSOME }\end{array}$ & $\begin{array}{l}\text { Population ICSI } \\
\text { Fréquence moyenne des } \\
\text { anomalies parmi les } \\
\text { spermatozoïdes étudiés } \\
\quad(n=625)\end{array}$ & $\begin{array}{l}\text { Population témoin } \\
\text { Fréquence moyenne des } \\
\text { anomalies parmi les } \\
\text { spermatozoïdes étudiés } \\
\qquad(\mathrm{n}=125)\end{array}$ & $\mathrm{p}$ \\
\hline \% de spermatozoïdes anormaux & $70,68 \pm 16,10$ & $46,40 \pm 6,06$ & $\underset{*}{<0.0001}$ \\
\hline Vacuoles $(\%)$ & $53,44 \pm 18,58$ & $34,40 \pm 9,21$ & $0.0001^{*}$ \\
\hline Base (\%) & $24,32 \pm 11,94$ & $16,80 \pm 5,21$ & NS \\
\hline Reste cytoplasmique (\%) & $13,60 \pm 14,52$ & $2,40 \pm 2,19$ & $0.0004^{*}$ \\
\hline Pièce intermédiaire irrégulière (\%) & $5,76 \pm 5,04$ & $1,60 \pm 3,56$ & NS \\
\hline Anomalie régionale (\%) & $4,00 \pm 4,32$ & $0,80 \pm 1,79$ & NS \\
\hline Tête amincie (\%) & $1,60 \pm 4,16$ & $0,80 \pm 1,79$ & NS \\
\hline Tête allongée (\%) & $0,16 \pm 0,80$ & 0 & \multirow{8}{*}{ NS } \\
\hline Contours irréguliers (\%) & $0,16 \pm 0,80$ & & \\
\hline Microcéphale (\%) & \multirow{6}{*}{0} & \multirow{6}{*}{0} & \\
\hline Macrocéphale (\%) & & & \\
\hline Têtes multiples (\%) & & & \\
\hline Pièce intermédiaire grêle (\%) & & & \\
\hline Angulation (\%) & & & \\
\hline Anomalie du flagelle (\%) & & & \\
\hline
\end{tabular}

* : significatif. NS : non significatif.

Tableau 5 : Comparaison des paramètres morphologiques évalués en MSOME en fonction de l'issue des tentatives d'ICSI.

\begin{tabular}{|c|c|c|c|c|}
\hline \multicolumn{2}{|c|}{ Détail des anomalies en MSOME } & "Grossesse » & "Pas de grossesse » & $\mathbf{p}$ \\
\hline \multicolumn{2}{|c|}{$\%$ de spermatozoïdes anormaux } & $76.7 \pm 16.3$ & $68.8 \pm 16.0$ & NS \\
\hline \multicolumn{2}{|c|}{ Tête allongée (\%) } & 0 & 0 & NS \\
\hline \multicolumn{2}{|c|}{ Tête amincie (\%) } & $4.8 \pm 7.6$ & $0.8 \pm 1.6$ & NS \\
\hline \multicolumn{2}{|c|}{ Microcéphale (\%) } & 0 & 0 & NS \\
\hline \multicolumn{2}{|c|}{ Macrocéphale (\%) } & 0 & 0 & NS \\
\hline \multicolumn{2}{|c|}{ Tête multiple (\%) } & 0 & 0 & NS \\
\hline \multicolumn{2}{|c|}{ Contours irréguliers (\%) } & 0 & $0.4 \pm 0.8$ & NS \\
\hline \multicolumn{2}{|c|}{ Anomalie régionale (\%) } & $4.8 \pm 4.8$ & $3.6 \pm 4.4$ & NS \\
\hline \multicolumn{2}{|c|}{ Base anormale (\%) } & $24.8 \pm 12.8$ & $24.4 \pm 12.0$ & NS \\
\hline \multirow{3}{*}{ Vacuoles } & Présence (\%) & $56.8 \pm 23.2$ & $52.4 \pm 17.6$ & NS \\
\hline & Nombre moyen & $6.8 \pm 2.0$ & $7.2 \pm 1.6$ & NS \\
\hline & Surface moyenne (\%) & $36.0 \pm 25.6$ & $34.8 \pm 24.0$ & NS \\
\hline \multicolumn{2}{|c|}{ Reste cytoplasmique (\%) } & $38.0 \pm 22.0$ & $25.2 \pm 17.6$ & NS \\
\hline \multicolumn{2}{|l|}{ Angulation (\%) } & 0 & 0 & NS \\
\hline \multicolumn{2}{|c|}{ Pièce intermédiaire grêle (\%) } & 0 & 0 & NS \\
\hline \multicolumn{2}{|c|}{ Pièce intermédiaire irrégulière (\%) } & $12.0 \pm 9.2$ & $10.0 \pm 13.2$ & NS \\
\hline \multicolumn{2}{|c|}{ Flagelle écourté (\%) } & 0 & 0 & NS \\
\hline \multicolumn{2}{|c|}{ Flagelle enroulé (\%) } & 0 & 0 & NS \\
\hline \multicolumn{2}{|c|}{ Flagelle multiple (\%) } & 0 & 0 & NS \\
\hline \multicolumn{2}{|c|}{ Flagelle absent (\%) } & 0 & 0 & NS \\
\hline \multicolumn{2}{|c|}{ Flagelle irrégulier (\%) } & 0 & 0 & NS \\
\hline
\end{tabular}

Valeur moyenne \pm écart-type ; NS = non significatif. 


\begin{tabular}{|c|c|}
\hline SPERMATOZOIDE & Normal \\
\hline \multirow{8}{*}{ TETE } & Allongée \\
\hline & Amincie \\
\hline & Microcéphale \\
\hline & Macrocéphale \\
\hline & Multiple \\
\hline & Contours irréguliers \\
\hline & Anomalie régionale \\
\hline & Base \\
\hline \multirow{2}{*}{ VACUOLES } & Nombre \\
\hline & $\%$ Surface \\
\hline \multirow{4}{*}{$\begin{array}{c}\text { PIECE } \\
\text { INTERMEDIAIRE }\end{array}$} & Reste cytoplasmique \\
\hline & Grêle \\
\hline & Angulation \\
\hline & Irrégulier \\
\hline \multirow{5}{*}{$\begin{array}{c}\text { FLAGELLE } \\
\text { PIECE PRINCIPALE }\end{array}$} & Absent \\
\hline & Ecourté \\
\hline & Irrégulier \\
\hline & Enroulé \\
\hline & Multiple \\
\hline
\end{tabular}

Figure 1 : Grille de lecture proposée en MSOME.

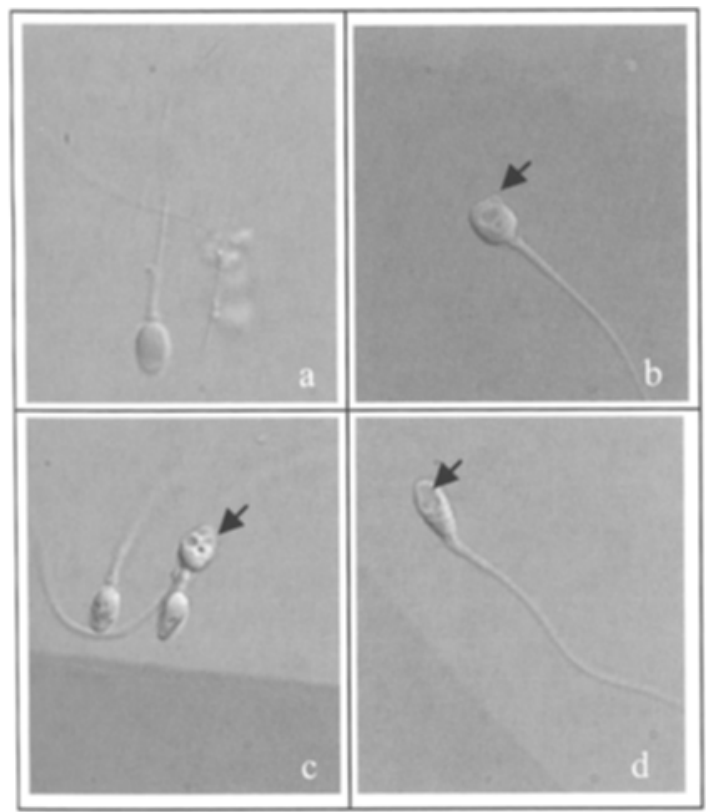

\begin{tabular}{|c|c|c|c|}
\hline \multicolumn{3}{|c|}{ Critères de la grille de lecture de Bartoov } & Adaptation des critères \\
\hline \multirow{7}{*}{ Noyau } & \multirow{6}{*}{ Forme } & Microcéphale & Inchangé \\
\hline & & Macrocéphale & Inchangé \\
\hline & & Aminci $(1<2,9 \mu \mathrm{M})$ & Inchangé \\
\hline & & Elargi $(1>3,7 \mu \mathrm{M})$ & Intégré au critere a Macroctphale " \\
\hline & & Count $(\mathrm{L}<4,2 \mu \mathrm{M})$ & Integré au critère \& Microcéphale \$ \\
\hline & & Anomalie Régionale & Inchangé \\
\hline & Chromatine & Surface Vacuoles $>4 \%$ & Detaille \\
\hline \multirow{3}{*}{\multicolumn{2}{|c|}{ Acrosome }} & Absence & \multirow{3}{*}{$\begin{array}{l}\text { Structure peu visible et évaluation du } \\
\text { taux d'anomalie impossible }\end{array}$} \\
\hline & & Particl & \\
\hline & & Vésiculé & \\
\hline \multirow{2}{*}{\multicolumn{2}{|c|}{ Cape Post-Acrosomique }} & Absence & \multirow{2}{*}{$\begin{array}{l}\text { Transforme en critère « Base } \\
\text { Anormale \# }\end{array}$} \\
\hline & & Vesiculee & \\
\hline \multirow{3}{*}{\multicolumn{2}{|c|}{ Cou }} & Angulation & Inchange \\
\hline & & Anomalie & $\begin{array}{l}\text { Intégré dans le critère « Pièce } \\
\text { Intermédiaire Imégulière : }\end{array}$ \\
\hline & & Reste Cytoplasmique & Inchangé \\
\hline \multirow{5}{*}{\multicolumn{2}{|c|}{ Flagelle }} & Absence & Inchangé \\
\hline & & Enroulé & Inchangé \\
\hline & & Cassé & Integré dans « Flagelle Court * \\
\hline & & Multiple & Inchange \\
\hline & & Count & Inchange \\
\hline \multirow{3}{*}{\multicolumn{2}{|c|}{ Mitechendrie }} & Absence & \multirow{3}{*}{ Structure non vue } \\
\hline & & Partiel & \\
\hline & & Désorganisation & \\
\hline
\end{tabular}

Figure 2 : Grille de lecture publiée par Bartoov et adaptations proposées.
Figure 3 : Exemples d'anomalies spermatiques observées en MSOME (x4500).

Photo a: Spermatozoïde normal.

Photo b : "Anomalie régionale ": "extrusion ou invagination de la masse nucleaire chromatinienne " selon Bartoov + vacuoles.

Photo $c$ : "Contours irréguliers " tête présentant sur une majorité de sa surface des irrégularités à type d'extrusion ou d'invagination.

Photo $d$ : 1 vacuole de surface $40-50 \%$. 
Le pourcentage de formes anormales à fort grossissement est de $70,7 \%$ dans le groupe A contre $46,4 \%$ dans le groupe $B(p<0,0001)$. Les vacuoles, les anomalies de la base et les restes cytoplasmiques sont les anomalies les plus représentées dans les deux groupes. La présence de vacuoles concerne tous les patients, l'anomalie étant prédominante pour 24 des 25 spermes étudiés dans le groupe $A$ et pour les 5 spermes du groupe B. En moyenne $53,4 \%$ des spermatozoïdes injectables présentent une ou plusieurs vacuoles dans le groupe $A$ contre $34,4 \%$ dans le groupe $B(p=0,0001)$. Dans le groupe $A, 24,3 \%$ des spermatozoïdes présentent une base anormale et $13,6 \%$ un reste cytoplasmique contre respectivement $16,8 \%$ (non significativement différent) et $2,4 \%$ des spermatozoïdes $(p=0,0004)$ dans le groupe $B$.

Notons que dans les deux groupes, la très grande majorité des vacuoles visualisées en MSOME se situe au niveau de la partie antérieure de la tête spermatique, en regard de la région acrosomique.

\section{Evaluation de la morphologie spermatique en MSOME et issue des tentatives}

Concernant les caractéristiques morphologiques spermatiques évaluées à fort grossissement, on ne constate aucune différence entre les deux groupes " Grossesse " et "Pas de grossesse " (Tableau 5). En particulier, le pourcentage de formes normales, le nombre de spermatozoïdes vacuolés, la surface moyenne des vacuoles par rapport à la tête spermatique ou le nombre moyen de vacuoles par spermatozoïde vacuolé ne sont pas différents.

De plus, il n'existe aucune corrélation entre le pourcentage de formes normales en MSOME et le taux de fécondation (Rho $=-0.36, p=0.08$ ) ou la qualité embryonnaire évaluée par le score de Steer (Rho = $0.18, p=0.39$ ). Enfin, il n'existe pas de corrélation entre le pourcentage de spermatozoïdes vacuolés en MSOME et le taux de fécondation (Rho $=0.22, p=0.29$ ) ou la qualité embryonnaire évaluée par le score de Steer (Rho $=0.19, p=0.36)$.

\section{DISCUSSION}

\section{Mise au point technique}

La grille de lecture en MSOME de Bartoov, proposée dans son article princeps [5], est inspirée des travaux réalisés par son équipe en microscopie électronique et notamment de l'approche dite d'analyse ultramorphologique quantitative (QUM) [6]. Cependant, après des essais de cette grille de lecture avec des spermes témoins, certains paramètres nous ont paru difficilement applicables en MSOME du fait d'une ambiguïté sur les structures décrites et de l'absence d'iconographie précise publiée (atlas d'anomalies). Nous avons donc adapté certains critères, tout en nous inspirant de la classification de David [1,15], classification validée et utilisée par de nombreuses équipes.

Initialement, il nous a semblé difficile et subjectif d'évaluer visuellement la surface des vacuoles par rapport à la surface de la tête. En préalable à la mise au point de la grille, nous avons comparé l'évaluation visuelle et la mesure à l'aide du logiciel Histolab 5.11.1 (Microvision Instruments) sur des spermatozoïdes préalablement immobilisés. Avec l'entraînement, l'évaluation visuelle, «tridimensionnelle» grâce à la vis micrométrique et assistée d'un "gabarit en celluloïd», s'est avérée aussi précise que l'évaluation à l'aide du logiciel Histolab (données non présentées) et présente une plus grande faisabilité en pratique courante.

\section{Evaluation de la morphologie spermatique en MSOME}

Dans la population « ICSI ", le pourcentage de formes effectivement normales en MSOME parmi les spermatozoïdes injectables varie de 0 à $56 \%$, avec une moyenne de $29,3 \%$. Dans l'étude prospective de Bartoov et al. [5] qui concernait 100 couples pris en charge en ICSI pour lesquels ils avaient examiné 100 spermatozoïdes mobiles non utilisés pour l'ICSI par échantillon, le pourcentage moyen de formes normales de spermatozoïdes était de $3,3 \%$ et variait de 0 à $38 \%$. Notons que dans cette étude, seuls les spermatozoïdes présentant une malformation sévère de la tête clairement identifiable à faible grossissement (tête d'épingle, amorphe, ronde, fuselée ou multiple) n'avaient pas été analysés en MSOME. Dans notre travail, la sélection effectuée au faible grossissement préalablement à l'observation en MSOME explique probablement les différences observées. Dans l'étude de Boughali et al. [10] qui concernait 52 couples pris en charge en FIV ou miFIV-milCSI pour échecs d'insémination intra-utérine, 6190 spermatozoïdes provenant de spermes normaux ou sub-normaux ont été analysés, sans présélection à faible grossissement, et le pourcentage moyen de formes normales était de 8,0\%.

Dans notre travail, l'anomalie prédominante pour 24 des 25 spermes étudiés est la présence de vacuoles. En moyenne, $53,4 \%$ de spermatozoïdes injectables présentent une ou plusieurs vacuoles. Ce chiffre est un peu inférieur à ceux de Boughali et al. [10] et Bartoov et al. [5] qui retrouvaient respectivement $86,6 \%$ et $73,2 \%$ de spermatozoïdes vacuolés, ce qui est probablement lié à notre présélection des spermatozoïdes à faible grossissement. Les deuxième et troisième anomalies les plus fréquemment rencontrées sont une base anormale $(24,3 \%$ des spermatozoïdes injectables observés) et la présence d'un reste cytoplasmique 
(13,6\% des spermatozoïdes injectables observés), confirmant ainsi les observations de Boughali et al. [10].

Dans cette population présélectionnée à faible grossissement, nous n'avons observé ni microcéphale, ni macrocéphale, ni tête multiple, ni angulation, ni pièce intermédiaire grêle, ni aucune anomalie de la pièce principale du flagelle.

Au total, plus de $70 \%$ des spermatozoïdes injectables dans des spermes de patients pris en charge en ICSI sont donc anormaux au fort grossissement. Ce chiffre très élevé pose la question des conséquences possibles de ces anomalies sur la fécondance des spermatozoïdes, de leur signification, ainsi que de l'aptitude de ces spermatozoïdes à permettre le développement embryonnaire. Or, les chiffres retrouvés dans la population de 5 spermes témoins (en moyenne $46,4 \%$ de spermatozoïdes anormaux dont $34,4 \%$ de spermatozoïdes vacuolés) sont comparables à ceux de certains spermes pathologiques de patients pris en charge en ICSI, et pourraient faire penser que certaines anomalies visualisées en MSOME ne sont que des variants phénotypiques. Néanmoins, les pourcentages de spermatozoïdes anormaux, de spermatozoïdes vacuolés et de spermatozoïdes présentant un reste cytoplasmique sont significativement plus élevés dans la population ICSI que dans la population témoin. Malgré les limites liées au faible effectif de notre série de spermes témoins, ces résultats suggèrent donc que certaines anomalies visualisées en MSOME auraient bien une valeur pathologique, hypothèse qu'il sera nécessaire de contrôler par l'étude d'un groupe témoin plus conséquent.

\section{Corrélation entre les paramètres conventionnels et l'observation en MSOME}

Contrairement à Bartoov et al. [5] et Boughali et al. [10] qui ne retrouvaient pas de corrélation entre la morphologie fine et l'analyse morphologique selon les critères de l'OMS, il existe dans notre travail une corrélation entre les paramètres morphologiques conventionnels évalués après migration le jour de la tentative et l'observation en MSOME (données non présentées). Cette différence est peut-être expliquée par notre choix de n'observer au MSOME que des spermatozoïdes " injectables », donc mobiles et indemnes d'anomalies morphologiques grossières.

\section{Evaluation de la morphologie spermatique en MSOME et issue des tentatives d'ICSI}

Dans ce travail, nous n'avons pas retrouvé de différence significative entre les 2 groupes "Grossesse " et « Pas de grossesse " concernant les caractéristiques morphologiques en MSOME, y compris sur la fréquence des vacuoles. Nous n'avons pas non plus mis en évidence de corrélation entre les pourcentages de formes anormales ou de spermatozoïdes vacuolés et les taux de fécondation, les taux de clivage et la qualité embryonnaire. Ces résultats sont en désaccord avec ceux de Bartoov et al. [5] qui rapportent une corrélation positive entre le pourcentage de formes normales et le taux de fécondation, ainsi qu'une corrélation entre le critère " noyau normal " (forme normale + absence de vacuoles) avec le taux de fécondation et l'obtention d'une grossesse dans une population.

Exceptée la présence d'un reste cytoplasmique qui semble être de mauvais pronostic au-delà de $25 \%$ des spermatozoïdes injectables, aucune autre anomalie ne parait présenter de valeur seuil au dessus de laquelle on n'observe pas de grossesse (données non présentées).

Contrairement à Bartoov et al. qui rapportaient qu'en dessous de $20 \%$ de spermatozoïdes à noyau normal aucune grossesse n'était obtenue [5], dans notre étude trois des six grossesses ont été obtenues avec des spermes présentant respectivement 4,12 et $16 \%$ de formes spermatiques normales, après présélection à faible grossissement. Ces spermes présentaient en outre en moyenne $72 \%$ de spermatozoïdes vacuolés. Dans cette série, les vacuoles ne semblent donc pas avoir de signification péjorative pour l'obtention d'une grossesse.

L'ensemble de ces résultats doit bien sûr être validé sur un plus grand nombre de patients.

\section{Perspectives}

Ce travail préliminaire nous a conduit à soulever plusieurs questions.

Tout d'abord, on peut s'interroger sur le support « anatomique " et la signification pathologique des anomalies visibles en MSOME, et en particulier des vacuoles " nucléaires » incriminées dans les échecs d'ICSI par d'autres auteurs [3, 7, 9, 21]. Nous nous sommes en effet demandé si les vacuoles visualisées en MSOME correspondaient exclusivement à de véritables vacuoles nucléaires ou également à des bulles acrosomiques. A plusieurs reprises, nos observations de ces vacuoles allaient dans le sens d'une origine multiple. En microscopie électronique les vacuoles nucléaires, résultats d'une condensation inégale de la chromatine, sont bien documentées [37] mais peu fréquentes et en général peu nombreuses et de petite taille. Ceci est à opposer aux bulles acrosomiques, liées à une migration anormale des vésicules golgiennes lors de la formation de l'acrosome, et qui sont par contre assez fréquentes. Ceci est conforté par le fait que la très grande majorité des vacuoles visualisées en MSOME se situe au niveau de la partie 
antérieure de la tête spermatique, en regard de la position de l'acrosome, et non pas à la base de la tête.

Ensuite, la question de la relation phénotype-génotype, donc de la possible corrélation entre la morphologie spermatique et l'intégrité génétique, est devenue une question particulièrement pertinente avec l'avènement de l'ICSI. Plusieurs études de caryotypes de spermatozoïdes humains ne suggèrent aucune relation entre la morphologie et les anomalies chromosomiques numériques et structurales [2, 28]. Par contre, les résultats des études de cytogénétique moléculaire (FISH) sont contradictoires [31, 35], [11, 18]. Le risque chromosomique est mieux documenté dans les syndromes homogènes conduisant à des tératospermies monomorphes comme dans le cas de la macrocéphalie [19], de certains cas de globozoospermie [12, 27] ou certains cas d'anomalies flagellaires [30]. Mais en dehors de ces cas particuliers, la sélection d'un spermatozoïde normal diminue-t-elle le risque de transmettre une anomalie génétique ? De même, la présence d'une anomalie morphologique préjuge-t-elle du patrimoine génétique ? Une étude séduisante a été menée par Celik et al. [13] dans laquelle des spermatozoïdes d'hommes fertiles ont été évalués en FISH, associée à une évaluation morphologique en contraste de phase des mêmes spermatozoïdes. Ils retrouvent que les anomalies chromosomiques numériques sont présentes dans des têtes de toutes formes et tailles, que des spermatozoïdes disomiques ou diploïdes ont parfois une morphologie strictement normale et que certains spermatozoïdes morphologiquement anormaux ont un contenu chromosomique normal.

Enfin, aucune étude prospective randomisée ne comparant IMSI et ICSI classique n'a été publiée, et les indications précises de la technique d'observation et de sélection à fort grossissement des spermatozoïdes ne sont pas validées. Sans randomisation, Bartoov et al. [3] puis Hazout et al. [21] ont comparé, chez respectivement 24 et 125 couples inclus après respectivement au moins 5 et 2 échecs d'ICSI classique, les issues des cycles d'IMSI avec les issues des tentatives d'ICSI classiques précédentes. D'autres publications retrouvent des résultats extrêmement prometteurs avec une augmentation des taux de grossesse évolutive et une diminution des taux de fausse-couche spontanée lorsque le cycle de microinjection se fait avec des spermatozoïdes morphologiquement sélectionnés à fort grossissement $[4,8]$. Cependant, elles présentent toutes des biais méthodologiques liés à la définition des groupes contrôles. Des essais prospectifs randomisés multicentriques en double-aveugle sembleraient maintenant nécessaires afin de conclure définitivement sur l'intérêt clinique et les indications de la méthode de sélection des spermatozoïdes à fort grossissement en ICSI.

Ainsi, à l'heure où de nombreuses équipes portent un intérêt croissant à la méthode d'observation et de sélection à fort grossissement des spermatozoïdes développée par Bartoov et al., nous avons souhaité réaliser un travail d'évaluation préalable de la technique. Dans notre courte série d'ICSI d'indications hétérogènes, nous avons retrouvé des fréquences très élevées d'anomalies (supérieures à $70 \%$ ), et notamment de vacuoles nucléaires. Nous n'avons mis en évidence aucune valeur prédictive de la morphologie des spermatozoïdes observés à fort grossissement sur les taux de fécondation, la qualité embryonnaire ou l'issue de la tentative d'ICSI. Cependant, les perspectives d'utilisation de cette nouvelle technique sont nombreuses, à la fois comme outil dans la compréhension des mécanismes physiopathologiques et en thérapeutique par la possible amélioration des taux de grossesse en ICSI dans certaines indications.

\section{RÉFÉRENCES}

1. AUGER J., EUSTACHE F. : Standardisation de la classification morphologique des spermatozoïdes humains selon la méthode de David modifiée. Andrologie, 2000, 10 : 358-373.

2. BALKAN W., MARTIN R.H. : Chromosome segregation into the spermatozoa of two men heterozygous for different reciprocal translocations. Hum. Genet., 1983, $63: 345-348$.

3. BARTOOV B., BERKOVITZ A., ELTES F. : Selection of spermatozoa with normal nuclei to improve the pregnancy rate with intracytoplasmic sperm injection. N. Engl. J. Med., 2001, 345 : 1067-1068.

4. BARTOOV B., BERKOVITZ A., ELTES F. et al. : Pregnancy rates are higher with intracytoplasmic morphologically selected sperm injection than with conventional intracytoplasmic injection. Fertil. Steril., 2003, $80: 1413-1419$.

5. BARTOOV B., BERKOVITZ A., ELTES F. et al. : Real-time fine morphology of motile human sperm cells is associated with IVF-ICSI outcome. J. Androl., 2002, $23: 1-8$.

6. BARTOOV B., ELTES F., REICHART M. et al. : Quantitative ultramorphological (QUM) analysis of human sperm : diagnosis and management of male infertility. Arch. Androl., 1999, 42: 161-177.

7. BERKOVITZ A., ELTES F., ELLENBOGEN A. et al. : Does the presence of nuclear vacuoles in human sperm selected for ICSI affect pregnancy outcome ? Hum. Reprod., 2006, 21: 1787-1790.

8. BERKOVITZ A., ELTES F., LEDERMAN H. et al. : How to improve IVF-ICSI outcome by sperm selection. Reprod. Biomed. Online, 2006, 12 : 634-638.

9. BERKOVITZA., ELTES F., YAARI S. et al. : The morphological normalcy of the sperm nucleus and pregnancy rate of intracytoplasmic injection with morphologically selected sperm. Hum. Reprod., 2005, 20 : 185-190.

10. BOUGHALI H., WITTEMER C., VIVILLE S. : Intérêt de l'analyse de la morphologie fine et de la qualité nucléaire 
des spermatozoïdes dans le cadre des techniques d'AMP. Andrologie, 2006, $16:$ 38-45.

11. CALOGERO A.E., DE PALMA A., GRAZIOSO C. et al. Aneuploidy rate in spermatozoa of selected men with abnormal semen parameters. Hum. Reprod., 2001, $16: 1172-1179$.

12. CARRELL D.T., WILCOX A.L., UDOFF L.C., THORP C., CAMPBELL B. : Chromosome 15 aneuploidy in the sperm and conceptus of a sibling with variable familial expression of round-headed sperm syndrome. Fertil. Steril., 2001, 76 : 1258-1260.

13. CELIK-OZENCI C., JAKAB A., KOVACS T. et al. : Sperm selection for ICSI: shape properties do not predict the absence or presence of numerical chromosomal aberrations. Hum. Reprod., 2004, 19 : 2052-2059.

14. COETZEE K., KRUGE T.F., LOMBARD C.J. : Predictive value of normal sperm morphology: a structured literature review. Hum. Reprod. Update, 1998, 4 : 73-82.

15. DAVID G., BISSON J.P., CZYGLIK F. et al. : Anomalies morphologiques du spermatozoïde humain. Propositions pour un système de classification. J. Gynecol. Obstet. Biol. Reprod., 1975, Suppl. 1 : 17-36.

16. DE VOS A., VAN DE VELDE H., JORIS $H$. et al. : Influence of individual sperm morphology on fertilization, embryo morphology, and pregnancy outcome of intracytoplasmic sperm injection. Fertil. Steril., 2003, $79: 42-48$.

17. DEVILLARD F., METZLER-GUILLEMAIN C., PELLETIER R. et al. : Polyploidy in large-headed sperm : FISH study of three cases. Hum. Reprod., 2002, 17 : 1292-1298.

18. GOLE L.A., WONG P.F., NG P.L. et al. : Does sperm morphology play a significant role in increased sex chromosomal disomy ? A comparison between patients with teratozoospermia and OAT by FISH. J. Androl., 2001, 22 : 759763.

19. GUTHAUSER B., VIALARD F., DAKOUANE M. et al. : Chromosomal analysis of spermatozoa with normal-sized heads in two infertile patients with macrocephalic sperm head syndrome. Fertil. Steril., 2006, $85: 750$ e5-750 e7.

20. GUZICK D.S., OVERSTREET J.W., FACTOR-LITVAK P. et al. : Sperm morphology, motility, and concentration in fertile and infertile men. N. Engl. J. Med., 2001, 345 : 1388-1393.

21. HAZOUTA., DUMONT-HASSAN M., JUNCAA.M., COHEN BACRIE P., TESARIK J. : High-magnification ICSI overcomes paternal effect resistant to conventional ICSI. Reprod. Biomed. Online, 2006, $12: 19-25$.

22. KILANI Z., ISMAIL R., GHUNAIM S. et al. : Evaluation and treatment of familial globozoospermia in five brothers. Fertil. Steril., 2004, 82 : 1436-1439.

23. KRUGER T.F., ACOSTAA.A., SIMMONS K.F. et al. : Predictive value of abnormal sperm morphology in in vitro fertilization. Fertil. Steril., 1988, $49: 112-117$.

24. KRUGER T.F., COETZEE K. : The role of sperm morphology in assisted reproduction. Hum. Reprod. Update, 1999, 5 : 172-178.

25. LEE R.K., HOU J.W., HO H.Y. et al. : Sperm morphology analysis using strict criteria as a prognostic factor in intrauterine insemination. Int. J. Androl., 2002, 25 : 277-280.

26. LUNDIN K., SODERLUND B., HAMBERGER L. : The relationship between sperm morphology and rates of fertilization, pregnancy and spontaneous abortion in an in vitro fertilization/intracytoplasmic sperm injection programme. Hum. Reprod., 1997, 12 : 2676-2681.

27. MARTIN R.H., GREENE C., RADEMAKER A.W. : Sperm chromosome aneuploidy analysis in a man with globozoospermia. Fertil. Steril., 2003, 79 Suppl 3 : 16621664.

28. MARTIN R.H., RADEMAKER A. : The relationship between sperm chromosomal abnormalities and sperm morphology in humans. Mutat. Res., 1988, 207 : 159-164.

29. MASHIACH R., FISCH B., ELTES F. et al. : The relationship between sperm ultrastructural features and fertilizing capacity in vitro. Fertil. Steril., 1992, 57 : 1052-1057.

30. RIVES N., MOUSSET-SIMEON N., MAZURIER S., MACE B.: Primary flagellar abnormality is associated with an increased rate of spermatozoa aneuploidy. J. Androl., 2005, 26 : 61-69.

31. RIVES N., SAINT CLAIRA., MAZURIER S. et al. : Relationship between clinical phenotype, semen parameters and aneuploidy frequency in sperm nuclei of 50 infertile males. Hum. Genet., 1999, $105:$ 266-272.

32. STEER C.V., MILLS C.L., TAN S.L., CAMPBELL S., EDWARDS R.G. : The cumulative embryo score : a predictive embryo scoring technique to select the optimal number of embryos to transfer in an in vitro fertilization and embryo transfer programme. Hum. Reprod., 1992, 7 : 117-119.

33. TESARIK J., GRECO E., MENDOZA C. : Late, but not early, paternal effect on human embryo development is related to sperm DNA fragmentation. Hum. Reprod., 2004, 19 : 611-615.

34. VAN DER MERWE F.H., KRUGER T.F., OEHNINGER S.C., LOMBARD C.J. : The use of semen parameters to identify the subfertile male in the general population. Gynecol. Obstet. Invest., 2005, $59: 86-91$.

35. VIVILLE S., MOLLARD R., BACH M.L. et al. : Do morphological anomalies reflect chromosomal aneuploidies?: case report. Hum. Reprod., 2000, 15 : 2563-2566.

36. WORLD HEALTH ORGANIZATION : WHO Laboratory Manual for the Examination of Human Semen and Semen-Cervical Mucus Interaction, 1999, 4th edition.

37. ZAMBONI L. : The ultrastructural pathology of the spermatozoon as a cause of infertility : the role of electron microscopy in the evaluation of semen quality. Fertil. Steril., $1987,48: 711-734$.

Manuscrit reçu : juillet 2007 ; accepté juillet 2007.

Prix Master 2006. 


\begin{abstract}
Evaluation of Motile Sperm Organelle Morphology Examination in unselected In Vitro Fertilization with Intra-Cytoplasmic Sperm Injection
\end{abstract}

Nathalie SERMONDADE, François VIALARD, Marianne BERGERE, Ibrahim HAMMOUD, Patrick CAVELOT, Jacqueline SELVA, Martine ALBERT

Due to the growing interest in the method of highmagnification sperm observation and selection proposed for the specific indication of ICSI failure, the authors evaluated the technique in unselected ICSI.

The aim of this study was to evaluate the relevance of Motile Sperm Organelle Morphology Examination (MSOME) compared with usual selection performed in ICSI. In a series of conventionally selected sperm for ICSI, the number with an abnormal appearance on high magnification was determined and the predictive value of this parameter on ICSI outcome was assessed.

The study included 25 successive unselected ICSI attempts in the IVF Laboratory of Poissy Hospital (France). ICSI were performed according to usual protocols used in the laboratory. Twenty five motile spermatozoa of the migrated fraction, still available after ICSI, and "injectable" according to conventional morphology assessment in ICSI ("normal" or "as normal as possible" with magnification of $\times 200-400$ ) were assessed by MSOME (higher than $\times 4500$ ) and classified according to criteria adapted from Bartoov's work and taking into account David's sperm morphology classification. We compared the results of MSOME and ICSI results.

In this small series of ICSI with diverse indications, we found very high frequencies of abnormalities (more than $70 \%$ ), particularly nuclear vacuoles. No predictive value of the morphology of sperm assessed with high magnification (including vacuoles) was found for fertilization rate, embryo quality and ICSI outcome. In contrast with previous reports, pregnancies were obtained with very abnormal sperms. In this series of unselected ICSI, nuclear vacuoles do not seem to have a pejorative impact on pregnancy outcome.

This study raises several perspectives. It would be interesting to understand the "anatomical" basis for vacuoles observed with MSOME and their meaning. The question of the phenotype-genotype relation, i.e. the possible correlation between sperm morphology and genetic content could be investigated. Finally, a prospective analysis should be performed in clearly defined indications to validate the potential applications of the method for high-magnification sperm observation and selection.

Key-words: sperm morphology, high-magnification sperm observation and selection, MSOME, ICSI, IMSI, nuclear vacuoles 